connections. This study uses MR time resolved angiography to examine contrast circulation in a cohort of patients with Fontan circulation to optimise contrast injection protocol for CT. Methods Time to peak signal intensity was recorded using regions of interest on the aorta, the pulmonary arteries and the Fontan conduit on MR TWIST angiography images. Patients were stratified into groups according to ejection fraction, global longitudinal strain, indexed stroke volume and cardiac index to examine the effect on time to peak signal intensity.

Results 35 patients were included in the study. Mean time to peak contrast enhancement was $31 \mathrm{~s}$ in the thoracic aorta, 46s in the right pulmonary artery, 41s in the left pulmonary artery and $55 \mathrm{~s}$ in the Fontan conduit. Cardiac performance shows little relationship to peak vascular enhancement whether measured by ejection fraction, global longitudinal strain, stroke volume index and cardiac index.

Discussion This MRI data suggest that optimal timing for a single phase examination to show all the major vessels is around 55 seconds following start of contrast injection. In TWIST MR angiography the IV bolus is 4-5 seconds long. A longer bolus is required for CTA, around 20s, suggesting an additional delay will be required. Further work will be done to see is a single phase examination at 70 seconds is an ideal initial strategy, with targeted further imaging if unsuccessful.

\section{THORACIC AORTA AND PULMONARY ARTERY DIMENSIONS IN PATIENTS UNDERGOING ECG-GATED COMPUTED TOMOGRAPHY ANGIOGRAPHY}

${ }^{1}$ Andrew Gentle, ${ }^{1,2}$ Michelle C Williams, ${ }^{1,2}$ David E Newby, ${ }^{3,4}$ Giles Roditi. ' University of Edinburgh/British Heart Foundation Centre for Cardiovascular Science, Edinburgh, UK; ${ }^{2}$ Edinburgh Imaging facility QMRI, University of Edinburgh, Edinburgh, UK; ${ }^{3}$ Glasgow Clinical Research Imaging Facility, Queen Elizabeth University Hospital, Glasgow, UK; ${ }^{4}$ Glasgow University, Glasgow, UK

\subsection{6/heartjnl-2019-BSCI.5}

Introduction Normal values for vascular dimensions are derived from echocardiography or magnetic resonance imaging (MRI), with limited data available on electrocardiogram (ECG)-gated computed tomography (CT) angiography. This abstract assesses aortic and pulmonary artery diameters and the factors affecting them, in patients undergoing CT as part of the SCOT-HEART (Scottish COmputed Tomography of the HEART) trial.

Methods Vessel diameters were measured on CT coronary angiography (CTCA) in six locations: annulus, sinus of Valsalva (SOV), sinotubular junction (STJ), ascending and descending aorta at right pulmonary artery level, and main pulmonary artery (MPA). The SCOT-HEART database provided demographics and cardiovascular risk factor information.

Results Images of 1000 patients (mean age 58.9, 58\% male) were assessed. Amongst patients with normal coronary arteries and no history of hypertension, mean dimensions were: annulus short-axis $19 \pm 2 \mathrm{~mm}$, annulus long-axis $27 \pm 3$ $\mathrm{mm}$, SOV $31 \pm 4 \mathrm{~mm}$, STJ $27 \pm 3 \mathrm{~mm}$, ascending aorta $30 \pm 4$ $\mathrm{mm}$, descending aorta $22 \pm 2 \mathrm{~mm}$, and MPA $23 \pm 3 \mathrm{~mm}$. Male sex, height, and body surface area (BSA) correlated with increases in all dimensions $(\mathrm{p}<0.001)$. Age correlated with aortic dimensions only $(\mathrm{p}<0.03)$. Patients with normal coronary arteries on CTCA had smaller aortic dimensions $(\mathrm{p}<0.001)$. Patients with hypertension had larger MPA and aortic dimensions $(p<0.02)$, apart from at the annulus. Sex and BSA were independent predictors of all dimensions. Hypertension was an independent predictor of some aortic dimensions. Age was an independent predictor of all aortic dimensions, except the annulus.

Conclusion Aortic and pulmonary dimensions can be assessed on CTCA, with sex and BSA having an important impact on normal dimensions.

\section{COMPARISON CTCA DOSE AUDIT BETWEEN INITIAL SERVICE DEVELOPMENT AND SUBSEQUENT SERVICE ESTABLISHMENT PHASE FOR A DGH CTCA SERVICE}

Vilim Kalamar, Peter Chapman, Julian Elford, Jade Fleet, Alison Wright, Zoe Crawley. Department of Radiology Hampshire Hospitals NHS Foundation Trust, Winchester, UK

\subsection{6/heartjnl-2019-BSCI.6}

Introduction The delivery of computed tomography coronary angiography (CTCA) services is variable but underprovided across the UK. Hampshire hospitals foundation trust (HHFT) is trying to contribute to this necessary national increase in CTCA provision. The aim of this poster presentation is presentation of our initial ionizing radiation dose audits for governance and to share issues we faced locally which might be relevant to other sites starting similar services.

Methods Retrospective analysis of all patients attending HHFT for CTCA at HHFT was obtained by interrogation of the Computerized Radiology Information System (CRIS) database between 2013 and April 2018, then re-audited between May 2018 and February 2019.The total Dose-Length Product (DLP) units ( $\mathrm{mGycm}$ ) and heart rate at time of acquisition were identified allowing comparison between the initial service development \& second service establishment phase.

Results The median total DLP was 113.11 during initial service development (January 2013 - April 2018 phase). The median total DLP was 192.91 during service establishment phase (May 2018 - February 2019) Median DLP became higher in establishment phase.

Conclusion Initial success of the service along with initial tight patient selection led to a lower first round DLP. Subsequent appointment of an Electrophysiology Cardiology Consultant and increasing awareness of service led to evolving referral patterns with more atrial fibrillation (AF) ablation scans requested. Identifying this in the second round of audit has led to discussions with referrers \& establishment of routine DLP recording onto CRIS

\section{RADIATION DOSES OF CT CORONARY ANGIOGRAMS DONE AT SOUTHEND UNIVERSITY HOSPITAL; ONE YEAR AFTER THE 2017 NATIONAL SURVEY}

Mohammad Ali Khan, Harith Adil Al-Qaysi. Southend University Hospital, UK

\subsection{6/heartjnl-2019-BSCI.7}

Introduction In 2018, Southend University Hospital performed 846 CT coronary angiograms. We participated in the 2017 Prospective National Survey of coronary CT radiation. Our median radiation dose was $194 \mathrm{mGycm}$ compared to the national median of $209 \mathrm{mGycm}$. After the audit, 64 slice scanners with improved low contrast detectability were installed. These were powered by Adaptive Statistical Iterative 
Reconstruction (ASiR-V), which eliminated background noise with BMI calculated adjustments.

Methods All scans were prospectively gated with zero padding. BMI was calculated and radiation dose adjusted accordingly. We collected retrospective data for coronary CT Angiograms that excluded repeat scans due to technical errors as well as graft studies to ensure uniformity. Data gathered included patient demographics, body mass index and exam dose-length product (DLP).

Results A total of 110 coronary CTA exams performed 1 year after the survey were randomly selected for analysis in our study. The median BMI and exam DLP were $27.7 \mathrm{~kg} / \mathrm{m}^{2}$ and $138 \mathrm{mGycm}$, respectively. The median exam DLP in our previous study was $194 \mathrm{mGycm}$. Therefore, the median exam DLP using the newer machines and reconstruction technique is lower by $28.86 \%$ in comparison to the previous survey. In addition, we observed a trend of higher exam DLP with increasing BMI.

Conclusion Using the newer scanners in combination with ASiR-V at our hospital led to reduced radiation doses.

\section{COMPARISON OF THE ACCURACY OF FLASH VERSUS PROSPECTIVELY ACQUIRED CT CORONARY ANGIOGRAPHY}

Kate Liang, Mark Dayer, Oliver E Gosling. Department of Cardiology, Taunton and Somerset Hospital

\subsection{6/heartjnl-2019-BSCI.8}

Introduction Computed Tomography Coronary Angiography (CTCA) is an accurate and established diagnostic tool. The development of ECG triggered spiral acquisition (FLASH) has allowed reduction in radiation dose whilst still providing diagnostic information. We aimed to compare the accuracy of FLASH and prospective CTCA acquisitions within our CT service.

Method Retrospective analysis of demographic and outcome data was performed for 2857 scans over a 69 month period (Feb 2013-Nov 2018). Results of CTCA and Invasive Coronary Angiography (ICA) were compared for patients having had FLASH or prospective scan protocols.

Results 289 patients were identified as having had both CTCA and ICA. 55 (19\%) of these studies were FLASH, 173 (59.9\%) prospective, $2(0.7 \%)$ retrospective, 41 (14.2\%) calcium score only, and $18(6.2 \%)$ requiring $\geq 2$ contrast studies. [Results are reported FLASH vs Prospective] Diagnostic scans in $92.7 \%$ vs $93.6 \%$. Mean BMI $\left(\mathrm{Kgm}^{2}\right): 28(22-46)$ vs $30(18-46)$. Mean acquisition HR (bpm): 55 (48-70) vs 59 (41-166, median 59). Median dose metoprolol (mg): $5 \quad(0-30)$ vs 5 (0-50). Median DLP $(\mathrm{mGy} * \mathrm{~cm}): 79(49-351)$ vs $199(60-964)$. There was $58.2 \%$ agreement with FLASH studies and ICA versus $67.1 \%$ agreement with prospective acquisitions. This was not statistically significant $(p=0.26)$.

Conclusion Our data demonstrates that there is no statistically significant difference between FLASH and prospectively gated CTCA studies within our service. There is however a trend demonstrating that FLASH scans are not as accurate as prospectively gated scans although this is in a relatively small patient population. More studies with larger patient numbers and multi-centre analysis are recommended.

\section{DUAL ANTIPLATELET THERAPY TO INHIBIT MYOCARDIAL INJURY IN PATIENTS WITH HIGH-RISK CORONARY ARTERY PLAQUE: A RANDOMISED CONTROLLED TRIAL}

${ }^{1}$ Alastair J Moss, 'Marc R Dweck, 'Mhairi K Doris, '1Jack PM Andrews, 'Rong Bing, ${ }^{1}$ Rachael 0 Forsythe, ${ }^{1}$ Timothy R Cartlidge, ${ }^{1}$ Tania A Pawade, ${ }^{1}$ Marwa Daghem, ${ }^{2}$ Jennifer B Raftis, ${ }^{1,3}$ Michelle C Williams, ${ }^{1,3}$ Edwin JR van Beek, ${ }^{4}$ Laura Forsyth, ${ }^{4}$ Steff $C$ Lewis, ${ }^{4}$ Robert Lee, ${ }^{1,5}$ Anoop SV Shah, ${ }^{1,5}$ Nicholas L Mills, ${ }^{1,3}$ David E Newby, ${ }^{1,6}$ Philip D Adamson. ${ }^{1}$ British Heart Foundation Centre for Cardiovascular Science, University of Edinburgh, Edinburgh, UK; ${ }^{2}$ Medical Research Council Centre for Inflammation Research, University of Edinburgh, Edinburgh, UK; ${ }^{3}$ Edinburgh Imaging, Queen's Medical Research Institute University of Edinburgh, Edinburgh, UK; ${ }^{4}$ Edinburgh Clinical Trials Unit, University of Edinburgh, Edinburgh, UK; ${ }^{5}$ Usher Institute of Population Health Sciences and Informatics, University of Edinburgh, UK; ${ }^{6}$ Christchurch Heart Institute, University of Otago, Christchurch, New Zealand

\subsection{6/heartjnl-2019-BSCI.9}

Introduction High-risk coronary atherosclerotic plaque is associated with higher plasma troponin concentrations suggesting ongoing myocardial injury that may be a target for dual antiplatelet therapy. The aim of this study is to determine whether ticagrelor reduces high-sensitivity troponin I concentrations in patients with established coronary artery disease and high-risk coronary plaque using coronary 18F-fluoride positron emission tomography-computed tomography.

Methods In a randomised double-blind placebo-controlled trial, patients with multivessel coronary artery disease underwent coronary $18 \mathrm{~F}$-fluoride positron emission tomography-computed tomography and measurement of high-sensitivity cardiac troponin I and were randomised (1:1) to ticagrelor $90 \mathrm{mg}$ twice daily or matched placebo. The primary endpoint was troponin I concentration at 30 days in patients with increased coronary 18F-fluoride uptake.

Results In total, 202 patients were randomized and 191 met the pre-specified criteria for inclusion in the primary analysis. In patients with increased coronary 18F-fluoride uptake $(n=120 / 191)$ there was no evidence that ticagrelor had an effect on plasma troponin concentrations at 30 days (ratio of geometric means for ticagrelor versus placebo, 1.11, [95\% confidence interval 0.90 to 1.36 ], $\mathrm{p}=0.32$ ). Over 1 year, ticagrelor had no effect on troponin concentrations in patients with increased coronary 18F-fluoride uptake (ratio of geometric means, $0.86,95 \%$ confidence interval 0.63 to 1.17 , $\mathrm{p}=0.33)$.

Conclusion Dual antiplatelet therapy with ticagrelor does not reduce plasma troponin concentrations in patients with coronary $18 \mathrm{~F}$-fluoride uptake. This suggests that subclinical plaque thrombosis does not contribute to ongoing myocardial injury in this setting.

\section{CAC-RDS AND CAD-RDS AS A POTENTIAL TOOL TO BETTER CHARACTERISE CAD PREVALENCE, SEVERITY AND VARIATION WITHIN UK NICE CG95 COMPLIANT PATHWAYS}

Matthias Schmitt, Abid Ullah, Anna Reid, Craig Caswell, Chris Wong, Gavin Freeman, Melanie Greaves, Gaetano Nucifora. Manchester University NHS Foundation Trust, UK

\subsection{6/heartinl-2019-BSCI.10}

Background CAC-RADS and CAD-RADS were introduced to create a standardized method to communicate findings of coronary calcium scoring (CCS) and coronary CT angiography (cCTA) and to facilitate standardized clinical decision making 